\title{
Probability-Guaranteed Envelope-Constrained Filtering for Nonlinear Systems Subject to Measurement Outliers
}

\author{
Lifeng Ma, Zidong Wang, Jun Hu, and Qing-Long Han
}

\begin{abstract}
This paper deals with the recursive filtering problem for nonlinear time-varying stochastic systems subject to possible measurement outliers. In order to mitigate the effects from possible abnormal measurements, we construct a filter with a saturation constraint imposed on the innovations where the saturation level is adaptively determined according to the estimation errors. Two performance indices, namely, the finite-horizon $H_{\infty}$ specification and the envelope-constraint criterion with a prescribed probability, are put forward to describe the transient characteristics of the filtering error dynamics over a specified time interval. The purpose of the addressed problem is to design a filter capable of guaranteeing both the finite-horizon $H_{\infty}$ performance index and the probability-guaranteed envelope-constraint. Sufficient conditions are derived for the existence of the desired filter via certain convex optimization algorithms. Finally, an illustrative numerical example is proposed to demonstrate the effectiveness of the developed algorithm.
\end{abstract}

Index Terms-Nonlinear systems, measurement outliers, $H_{\infty}$ filtering, envelope-constraint in probability, finite-horizon filtering

\section{INTRODUCTION}

The past several decades have seen a surge of research interest on the filtering problems for stochastic systems and a multitude of filtering techniques have been reported in the literature, see e.g. [3], [5], among which the celebrated Kalman filtering (KF) algorithm has been playing a crucial role in the research areas of signal processing and stochastic control [12], [23], [31]. The KF algorithm serves as an optimal filtering technique in the sense of least mean square for linear models with Gaussian noises. Note that the performance of the standard KF algorithm would be significantly degraded when the system parameters are not perfectly known and/or the statistical information of the external noises is unavailable. Accordingly, alternative filtering approaches have been proposed to cope with the systems subject to uncertainty, nonlinearity and/or non-Gaussian noises. Examples include, but are not limited to, the robust KF algorithm [27], the extended KF (EKF) method [16], the set-valued filtering strategy [6] and the $H_{\infty}$ filtering scheme [22].

It is worth mentioning that, different from the KF algorithm which provides the optimal estimation in the sense of the least mean square, the $H_{\infty}$ approach aims to minimize the $H_{\infty}$ criterion quantizing the effects from the external disturbances to the estimation errors. Due

This work was supported in part by the National Natural Science Foundation of China under Grants 61773209, 61873148, 61933007 and 61673141, the Australian Research Council Discovery Project under Grant No. DP160103567, the Natural Science Foundation of Jiangsu Province of China under Grant BK20190021, the Six Talent Peaks Project in Jiangsu Province of China under Grant XYDXX-033, and the Alexander von Humboldt Foundation of Germany. (Corresponding author: Zidong Wang).

L. Ma is with the School of Automation, Nanjing University of Science and Technology, Nanjing 210094, China.

Z. Wang is with the College of Electrical Engineering and Automation, Shandong University of Science and Technology, Qingdao 266590, China. $\mathrm{He}$ is also with the Department of Computer Science, Brunel University London, Uxbridge, Middlesex, UB8 3PH, United Kingdom. (Email: Zidong. Wang@brunel.ac.uk)

J. Hu is with the Department of Mathematics, Harbin University of Science and Technology, Harbin 150080, China.

Q.-L. Han is with the School of Software and Electrical Engineering, Swinburne University of Technology, Melbourne, VIC 3122, Australia. to its robustness against parameter uncertainties as well as external disturbances, the $H_{\infty}$ filtering has captured an ever-increasing interest in both theoretical research and engineering applications, especially in the occasions when the statistical information of the disturbances is not available. So far, a rich body of literature has been available on the $H_{\infty}$ filtering problems and a number of techniques have been exploited that include the linear matrix inequality (LMI) approach, the Riccati equation method and the game theory strategy. Note that, in practical engineering, most systems are inevitably subject to timevarying parameters. Consequently, the $H_{\infty}$ filtering problems for time-varying systems over a finite horizon have drawn much research attention, see e.g. [10] for some recent results.

As far as the nonlinear systems are concerned, the corresponding $H_{\infty}$ filtering problem has been garnering a growing interest in recent years [11], [21], [22]. For instance, the $H_{\infty}$ filtering problem has been investigated in [22], where the underlying complex network is modeled by nonlinear functions constrained by known sector bounds. In particular, for general nonlinear systems, the existence of the corresponding $H_{\infty}$ filter can be cast into the solvability to certain Hamilton-Jacobi equation (HJE) or Hamilton-Jacobi inequality (HJI) [21]. Unfortunately, the technical difficulty in solving the HJE or HJI largely hinders the obtained results from being extended or applied. As such, some attention has recently been drawn to nonlinear filtering problems based on the traditional $H_{\infty}$ framework. A quintessential example is that, by borrowing the idea from EKF, the extended $H_{\infty}$ filter has been designed in [11] with its structure similar to the extended Kalman filter. Nevertheless, to the best of the authors' knowledge, the finite-horizon $H_{\infty}$ filtering problem for general nonlinear systems has not been sufficiently examined yet, which still remains as a challenging research issue.

On another research frontier, the envelope-constrained filtering (ECF) algorithm has recently stirred some research interest. The ECF algorithm aims to confine the output of filtering error (stimulated by a specified input) into a prescribed envelope that is determined by the desired output and the tolerance band. The ECF technique can find wide applications in a variety of engineering areas ranging from signal processing to digital communications [8], [10]. Up to now, several methodologies have been utilized in the literature to deal with the envelope-constrained filtering problems and some representative work can be summarized as follows. In an $H_{\infty}$ framework, the envelope-constrained filtering problem has been formulated in [30] and the solvability has been presented in terms of the feasibility of a finite dimensional minimax problem with linear inequality constraints. By resorting to the LMI approach, an infinite impulse response (IIR) filter has been designed in [24] that ensures that the system dynamics is bounded in a prespecified envelope. It should be pointed out that almost all the ECF-related results have been concerned with the linear time-invariant (LTI) systems, and very few results have been obtained with regard to the general nonlinear time-varying systems, which motivates us to shorten such a gap by conducting the current investigation.

It is widely recognized that the measurement outliers has now become a rather unavoidable phenomenon that might lead to deterioration of the estimation performance. Therefore, much effort 
has been devoted to the investigations of filtering/estimation issues subject to outliers, and several approaches have been proposed that can be categorized as follows. Within the Kalman filtering framework, the state estimation problems have been solved in [14] where the proposed algorithms are robust against the outliers. Recently, the moving horizon technique has been adopted in [1] with the purpose of estimating the states of LTI systems in the presence of measurement outliers. An observer has been designed in [2] for LTI system with measurements subjected to outliers whose effects are mitigated by introducing a saturated output injection. It is worth mentioning that, however, when the nonlinear time-varying systems are concerned, the corresponding results have been very much scattered, not to mention the case where the multiple performance requirements (i.e., $H_{\infty}$ specification and envelope-constraint) are simultaneously considered in a unified framework. It is, therefore, our aim in this paper to deal with the identified challenges by launching a major study on the socalled envelope-constrained $H_{\infty}$ filtering problem.

The main contributions of this paper can be highlighted as follows. i) The model of the system under investigation is comprehensive which is described by general nonlinear stochastic time-varying difference equation. ii) In order to mitigate the effects of possible measurement outliers, a mechanism of saturating innovations is adopted where the adaptive saturation level is recursively determined at each time step according to previous filtering errors. In comparison to those saturation mechanism with fixed levels, the proposed dynamical level can be adjusted adaptively along with the filtering accuracy and provide better performance. iii) To characterize the transient performances, two indices (i.e., $H_{\infty}$ specification and envelopeconstraint in probability) are defined over a finite horizon to depict dynamical behaviors from different perspectives. iv) The proposed algorithm is capable of guaranteeing the envelope-constraint with a predetermined probability (rather than the usual 100\% confidence). Such a probabilistically design method could provide much extra flexibility by relaxing certain stringent yet unnecessary performance constraints in real-world applications.

The rest of this paper is organized as follows. Section II formulates the probability-guaranteed envelope-constrained $H_{\infty}$ filtering problem for discrete-time nonlinear system subject to measurement outliers. The main results are presented in Section III where sufficient conditions for solvability of the addressed filtering problem are given in terms of recursive linear matrix inequalities (RLMIs). Section IV gives a numerical example and Section $\mathrm{V}$ outlines our conclusion.

Notation The notation used here is fairly standard except where otherwise stated. $\mathbb{R}^{n}$ denotes the $n$-dimensional Euclidean space, $\mathbf{1}_{n}$ denotes an $n$-dimensional column vector with all ones, $I_{n}$ and $0_{n}$ denote the identity matrix and zero matrix of $n$ dimensions, respectively. The notation $X \geq Y$ (respectively $X>Y$ ), where $X$ and $Y$ are symmetric matrices, means that $X-Y$ is positive semi-definite (respectively positive definite). The superscript " $\mathrm{T}$ " denotes the transpose. For a vector $a,\|a\|^{2}=a^{\mathrm{T}} a$ and for a scalar $b,|b|$ denotes the absolute value of $b$. $\operatorname{diag}\left\{F_{1}, F_{2}, \ldots, F_{n}\right\}$ denotes a block diagonal matrix whose diagonal blocks are given by $F_{1}, F_{2}, \ldots, F_{n}$. The notation $\operatorname{diag}_{n}\left\{A_{i}\right\}$ represents the block diagonal matrix $\operatorname{diag}\left\{A_{1}, A_{2}, \ldots, A_{n}\right\}$ and $\operatorname{col}_{n}\left\{x_{i}\right\}$ denotes the column vector $\left[\begin{array}{llll}x_{1}^{\mathrm{T}} & x_{2}^{\mathrm{T}} & \ldots & x_{n}^{\mathrm{T}}\end{array}\right]^{\mathrm{T}}$.

\section{Problem Formulation}

Consider the following nonlinear system defined on the horizon $[0, N]$ :

$$
\left\{\begin{aligned}
x_{k+1} & =f\left(x_{k}\right)+B_{k} \mu_{k}+\left(h\left(x_{k}\right)+E_{k} \mu_{k}\right) \omega_{k} \\
y_{k} & =g\left(x_{k}\right)+D_{k} \nu_{k} \\
z_{k} & =L_{k} x_{k}
\end{aligned}\right.
$$

where $x_{k} \in \mathbb{R}^{n_{x}}, y_{k} \in \mathbb{R}^{n_{y}}$ and $z_{k} \in \mathbb{R}$ represent, respectively, the system state, the measurement output and the signal to be estimated; $\mu_{k} \in l_{2}\left([0, N] ; \mathbb{R}^{n_{\mu}}\right)$ and $\nu_{k} \in l_{2}\left([0, N] ; \mathbb{R}^{n_{\nu}}\right)$ are the disturbance inputs; $\omega_{k} \in \mathbb{R}$ is a white Gaussian sequence with $\mathbb{E}\left\{\omega_{k}\right\}=0$ and $\mathbb{E}\left\{\omega_{k}^{2}\right\}=\alpha^{2} ; B_{k}, D_{k}, E_{k}$ and $L_{k}$ are known time-varying matrices with appropriate dimensions; and the nonlinear functions $f\left(x_{k}\right)$, $g\left(x_{k}\right)$ and $h\left(x_{k}\right)$ in system (1) are known and analytic everywhere over the finite horizon $[0, N]$. Without loss of generality, we assume that the output $z_{k}$ is a scalar so as to avoid unnecessarily complicated presentations, and the case when $z_{k}$ is a vector can be easily handled by applying the developed scheme.

Denoting $\hat{y}_{k} \triangleq g\left(\hat{x}_{k}\right)$ and $r_{k} \triangleq y_{k}-\hat{y}_{k}$, the filter to be designed in this paper is of the following form:

$$
\begin{aligned}
\hat{x}_{k+1} & =F_{k} \hat{x}_{k}+H_{k} \operatorname{Sat}_{\sigma_{k}}\left(y_{k}-\hat{y}_{k}\right) \\
& =F_{k} \hat{x}_{k}+H_{k} \operatorname{Sat}_{\sigma_{k}}\left(r_{k}\right), \quad \hat{x}_{0}=0
\end{aligned}
$$

where the nonlinear mapping $\operatorname{Sat}_{\sigma_{k}}(\cdot): \mathbb{R}^{n_{y}} \mapsto \mathbb{R}^{n_{y}}$ in filter (2) is a saturation function defined as follows:

$$
\operatorname{Sat}_{\sigma_{k}}\left(r_{k}\right) \triangleq\left[\begin{array}{c}
\operatorname{Sat}_{\sigma_{k}}^{(1)}\left(r_{k}\right) \\
\operatorname{Sat}_{\sigma_{k}}^{(2)}\left(r_{k}\right) \\
\vdots \\
\operatorname{Sat}_{\sigma_{k}}^{\left(n_{y}\right)}\left(r_{k}\right)
\end{array}\right]
$$

where $\operatorname{Sat}_{\sigma_{k}}^{(l)}\left(r_{k}\right) \triangleq \operatorname{sign}\left(r_{k}^{(l)}\right) \cdot \min \left\{\left|r_{k}^{(l)}\right|, \sigma_{k}\right\}$ with $r_{k}^{(l)}$ denoting the $l$-th entry of the vector $r_{k}$. Note that the saturation level $\sigma_{k}$ is time-varying which is determined adaptively at each time step by the following difference equation:

$$
\sigma_{k+1}=\lambda \sigma_{k}+\left(y_{k}-\hat{y}_{k}\right)^{\mathrm{T}} R_{k}\left(y_{k}-\hat{y}_{k}\right)
$$

where $\lambda \in[0,1)$ and $R_{k}$ is a predetermined positive definite matrix.

Remark 1: In filter (2), a purposely designed saturation function is adopted to mitigate the effects from possible measurement outliers by constraining the innovations (i.e., the differences between the measurement and estimated outputs) fed to the filter. It is worth mentioning that, different from those existing literature concerning saturation phenomenon where the saturation level is always assumed to be fixed [7], in this paper, we employ the iterative function (3) to determine the saturation level $\sigma_{k}$ by making use of the innovation information at corresponding time step. In specific, it is seen from (3) that when the innovation becomes smaller at time step $k$ (i.e., the estimation error becomes smaller), the saturation level $\sigma_{k+1}$ will become lower which indicates that the corresponding constraint imposed on innovation at time step $k+1$ will be more stringent. By introducing such a mechanism, the saturation level can be adjusted adaptively along with the filtering performances and the corresponding superiority will also be shown later in the simulation example.

For brevity of presentation, we denote $\phi_{k}(\cdot) \triangleq \mathbf{S a t}_{\sigma_{k}}(\cdot)$. Then, according to [26], there exists a diagonal matrix $\Lambda_{k}$ satisfying $0 \leq$ $\Lambda_{k} \leq I$ such that

$$
\left(\phi_{k}\left(r_{k}\right)-\Lambda_{k} r_{k}\right)^{\mathrm{T}}\left(\phi_{k}\left(r_{k}\right)-r_{k}\right) \leq 0
$$

where $\Lambda_{k} \triangleq \operatorname{diag}\left\{\varrho_{1 k}, \varrho_{2 k}, \ldots, \varrho_{n_{y} k}\right\}$ with $0 \leq \varrho_{i k} \leq 1$.

Remark 2: It should be mentioned that in most existing literature using the similar techniques to deal with saturation phenomenon, the matrix $\Lambda_{k}$ has been assumed to be fixed since the saturation level is fixed. In this paper, however, it can be known from (3) and (4) that $\Lambda_{k}$ should be appropriately selected to be a time-varying matrix as the saturation level $\sigma_{k}$ is time-varying. Actually, the value of the time-varying $\Lambda_{k}$ should be determined according to both values of $\sigma_{k}$ and $r_{k}$ at the corresponding time step. 
By using Taylor's expansion formula, we have

$$
\begin{aligned}
f\left(x_{k}\right) & =f\left(\hat{x}_{k}\right)+\Phi_{k}\left(x_{k}-\hat{x}_{k}\right)+\Sigma_{f k} \Delta_{f k}\left(x_{k}-\hat{x}_{k}\right) \\
g\left(x_{k}\right) & =g\left(\hat{x}_{k}\right)+\Psi_{k}\left(x_{k}-\hat{x}_{k}\right)+\Sigma_{g k} \Delta_{g k}\left(x_{k}-\hat{x}_{k}\right) \\
h\left(x_{k}\right) & =h\left(\hat{x}_{k}\right)+\Pi_{k}\left(x_{k}-\hat{x}_{k}\right)+\Sigma_{h k} \Delta_{h k}\left(x_{k}-\hat{x}_{k}\right)
\end{aligned}
$$

where $\Sigma_{f k}, \Sigma_{g k}$ and $\Sigma_{h k}$ are known scaling matrices, $\Delta_{f k}, \Delta_{g k}$ and $\Delta_{h k}$ are unknown matrices satisfying $\left\|\Delta_{f k}\right\| \leq 1,\left\|\Delta_{g k}\right\| \leq 1$ and $\left\|\Delta_{h k}\right\| \leq 1$, and $\Phi_{k}, \Psi_{k}$ and $\Pi_{k}$ are computed as follows:

$$
\Phi_{k}=\left.\frac{\partial f}{\partial x}\right|_{x=\hat{x}_{k}}, \quad \Psi_{k}=\left.\frac{\partial g}{\partial x}\right|_{x=\hat{x}_{k}}, \quad \Pi_{k}=\left.\frac{\partial h}{\partial x}\right|_{x=\hat{x}_{k}} .
$$

Remark 3: In (5), the terms $\Sigma_{f k} \Delta_{f k}\left(x_{k}-\hat{x}_{k}\right), \Sigma_{g k} \Delta_{g k}\left(x_{k}-\right.$ $\left.\hat{x}_{k}\right)$ and $\Sigma_{h k} \Delta_{h k}\left(x_{k}-\hat{x}_{k}\right)$ account for the truncation errors due to the neglected higher order terms in the Taylor series expansion of the corresponding nonlinear functions. Alternatively, $\Delta_{f k}, \Delta_{g k}$ and $\Delta_{h k}$ can also be illustrated as uncertain terms that are employed to describe the modeling errors in the matrices $\Phi_{k}, \Psi_{k}$ and $\Pi_{k}$, respectively. Moreover, it should be pointed out that the adoption of $\Delta_{f k}, \Delta_{g k}$ and $\Delta_{h k}$ will inevitably induces certain conservatism since the original nonlinear system is replaced by an approximated one in the filter design. Nevertheless, by appropriately choosing the scaling matrices $\Sigma_{f k}, \Sigma_{g k}$ and $\Sigma_{h k}$, we can largely reduce the conservatism of the proposed linearized model as well as the corresponding filtering approach. The readers are referred to [6] for a rigorous justification of the validity of such a representation for the linearization error.

By denoting $e_{k} \triangleq x_{k}-\hat{x}_{k}$, we acquire the following filtering error system:

$$
\left\{\begin{aligned}
e_{k+1}= & f\left(x_{k}\right)+B_{k} \mu_{k}+\left(h\left(x_{k}\right)+E_{k} \mu_{k}\right) \omega_{k} \\
& -F_{k} \hat{x}_{k}-H_{k} \phi_{k}\left(r_{k}\right) \\
= & f\left(\hat{x}_{k}\right)+\Phi_{k} e_{k}+\Sigma_{f k} \Delta_{f k} e_{k}+B_{k} \mu_{k} \\
& +\left(h\left(\hat{x}_{k}\right)+\Pi_{k} e_{k}+\Sigma_{h k} \Delta_{h k} e_{k}+E_{k} \mu_{k}\right) \omega_{k} \\
& -F_{k} \hat{x}_{k}-H_{k} \phi_{k}\left(r_{k}\right) \\
\tilde{z}_{k}= & L_{k} e_{k}
\end{aligned}\right.
$$

We are now ready to give the main objectives of this paper. It is our aim to design the filter parameters $F_{k}$ and $H_{k}$ in (2) such that the following two requirements are met simultaneously:

$R 1:\left(H_{\infty}\right.$ specification) Given $\gamma>0$ and $\Gamma>0$, the output $\tilde{z}_{k}$ of the filtering error system (6) satisfies

$$
\sum_{k=1}^{N} \mathbb{E}\left\{\left\|\tilde{z}_{k}\right\|^{2}\right\} \leq \gamma^{2} \sum_{k=1}^{N}\left\|\xi_{k}\right\|^{2}+\gamma^{2} e_{0}^{\mathrm{T}} \Gamma e_{0}
$$

for any nonzero $\xi_{k} \triangleq\left[\begin{array}{cc}\mu_{k}^{\mathrm{T}} & \nu_{k}^{\mathrm{T}}\end{array}\right]^{\mathrm{T}} \neq 0$.

$R 2$ : (Envelope-constraint in probability p) Under zero-initial condition, with the following input

$$
\xi_{k}^{\circ}= \begin{cases}\mathbf{1}, & k=0 \\ 0, & 1 \leq k \leq N\end{cases}
$$

the corresponding output $\tilde{z}_{k}^{\circ}$ of filtering error system (6) achieves

$$
\mathbb{P}\left\{\left|\tilde{z}_{k}^{\circ}-\varphi_{k}\right| \leq \chi_{k}\right\} \geq \mathbf{p}
$$

where $0<\mathbf{p}<1$ is a prespecified constant, and $\varphi_{k}$ and $\chi_{k}$ stand for, respectively, the desired output and the tolerance band of the output estimation error $\tilde{z}_{k}^{\circ}$.

Remark 4: For the time-varying nonlinear filtering error system (6), we are naturally interested in the transient behavior over a specified time interval. As a result, in this paper, two performance indices (i.e., the $H_{\infty}$ criterion and the envelope-constraint) are defined over the finite horizon $[0 \mathrm{~N}]$ to reflect the time-varying manner and characterize the transient dynamics of the addressed nonlinear system. Specifically, on one hand, the $H_{\infty}$ requirement in (7) is put forward to depict the robustness against the disturbances over the time interval $[0, N]$. On the other hand, the envelope-constraint in (9) is proposed to confine, at each time step $k$, the output estimation error within certain prespecified band (described by the desired output and tolerance band). It is worth mentioning that, although both requirements are proposed to describe the transient characteristics, they actually reflect the filtering error dynamics from different facets. The $H_{\infty}$ criterion concerns the disturbance attenuation level over the whole time interval $[0, N]$, whereas the envelope-constraint regards the dynamical behavior of the filtering error at each time instant $k$.

Remark 5: In real-world applications, it is usually impractical, if not impossible, to achieve the desired objectives with $100 \%$ confidence. Moreover, in engineering practice, it is unnecessary in many situations to require the performance indices to be satisfied with probability 1 . For example, the missile navigation/control specification might require that the standard deviation of the missile's altitude error should be less than 10 meters with an $80 \%$ probability. This gives rise to the so-called probability-guaranteed design issue whose objective is to attain the performance with a satisfactory chance [28]. Accordingly, instead of proposing the performance index with a hard bound [10], [18], in this paper, we aim to design the filter ensuring that the specified output $\tilde{z}_{k}^{\circ}$ is confined in the envelope band with certain pre-determined probability $\mathbf{p}$.

\section{MAin Results}

The following lemmas are useful in the establishment of our main results.

Lemma 1: (S-procedure [4]) Let $\psi_{0}(\cdot), \psi_{1}(\cdot), \ldots, \psi_{p}(\cdot)$ be quadratic functions of the variable $\varsigma \in \mathbb{R}^{n}: \psi_{j}(\varsigma) \triangleq \varsigma^{\mathrm{T}} X_{j} \varsigma(j=0, \ldots, p)$, where $X_{j}^{\mathrm{T}}=X_{j}$. If there exist $\epsilon_{1} \geq 0, \ldots, \epsilon_{p} \geq 0$ such that $X_{0}-\sum_{j=1}^{p} \epsilon_{j} X_{j} \leq 0$, then the following is true:

$$
\psi_{1}(\varsigma) \leq 0, \ldots, \psi_{p}(\varsigma) \leq 0 \rightarrow \psi_{0}(\varsigma) \leq 0 .
$$

Lemma 2: (Schur Complement Equivalence) Given constant matrices $\mathcal{S}_{1}, \mathcal{S}_{2}, \mathcal{S}_{3}$ where $\mathcal{S}_{1}=\mathcal{S}_{1}^{\mathrm{T}}$ and $0<\mathcal{S}_{2}=\mathcal{S}_{2}^{\mathrm{T}}$, then $\mathcal{S}_{1}+\mathcal{S}_{3}^{\mathrm{T}} \mathcal{S}_{2}^{-1} \mathcal{S}_{3}<0$ if and only if

$$
\left[\begin{array}{cc}
\mathcal{S}_{1} & \mathcal{S}_{3}^{\mathrm{T}} \\
\mathcal{S}_{3} & -\mathcal{S}_{2}
\end{array}\right]<0 \quad \text { or } \quad\left[\begin{array}{cc}
-\mathcal{S}_{2} & \mathcal{S}_{3} \\
\mathcal{S}_{3}^{\mathrm{T}} & \mathcal{S}_{1}
\end{array}\right]<0 .
$$

Lemma 3: [25] For any known matrix $\mathcal{G}>0$ and vector $b$ with appropriate dimensions, an ellipsoid $\mathcal{B}$ is defined by

$$
\mathcal{B} \triangleq\left\{z \mid(z-b)^{\mathrm{T}} \mathcal{G}(z-b) \leq 1\right\}
$$

where $z$ is a random variable. If, for any given $0<\mathbf{p}<1$, the following inequality

$$
\mathbb{E}\left\{(z-b)^{\mathrm{T}} \mathcal{G}(z-b)\right\} \leq 1-\mathbf{p}
$$

is true, then we have

$$
\mathbb{P}\{z \in \mathcal{B}\} \geq \mathbf{p} .
$$

\section{A. The $H_{\infty}$ specification}

For the convenience of subsequent derivation, we first give the following denotations:

$$
\begin{aligned}
& U_{k} \triangleq \frac{1}{2}\left(\Lambda_{k}+I\right), \quad \mathcal{D}_{k} \triangleq\left[\begin{array}{llll}
0 & D_{k}
\end{array}\right], \\
& \mathcal{I} \triangleq\left[\begin{array}{lllllll}
0 & 0 & 0 & 0 & 0 & 0 & I
\end{array}\right], \\
& \tilde{g}_{k} \triangleq\left[\begin{array}{lllllll}
0 & I & \mathcal{D}_{k} & 0 & 0 & \Sigma_{g k} & 0
\end{array}\right], \\
& \vartheta_{k} \triangleq \Delta_{f k} e_{k}, \delta_{k} \triangleq \Delta_{h k} e_{k}, \\
& \rho_{k} \triangleq \Delta_{g k} e_{k}, \phi_{k} \triangleq \phi_{k}\left(r_{k}\right),
\end{aligned}
$$




$$
\zeta_{k} \triangleq\left[\begin{array}{ll}
1 & e_{k}^{\mathrm{T}}
\end{array}\right]^{\mathrm{T}}
$$

where all the entries 0 and $I$ (namely, zero matrices and identity matrices) in the matrices throughout the paper are of suitable dimensions unless stated otherwise.

The filtering error dynamics (6) can be reformulated as

$$
\left\{\begin{aligned}
\zeta_{k+1}= & \hat{f}_{k} \zeta_{k}+\hat{B}_{k} \mu_{k}+\hat{\Sigma}_{f k} \vartheta_{k}-\hat{H}_{k} \phi_{k} \\
& +\left(\hat{h}_{k} \zeta_{k}+\hat{\Sigma}_{h k} \delta_{k}+\hat{E}_{k} \mu_{k}\right) \omega_{k} \\
\tilde{z}_{k}= & \mathcal{L}_{k} \zeta_{k}
\end{aligned}\right.
$$

where

$$
\begin{gathered}
\hat{f}_{k} \triangleq\left[\begin{array}{cc}
1 & 0 \\
f\left(\hat{x}_{k}\right)-F_{k} \hat{x}_{k} & \Phi_{k}
\end{array}\right], \quad \hat{h}_{k} \triangleq\left[\begin{array}{cc}
0 & 0 \\
h\left(\hat{x}_{k}\right) & \Pi_{k}
\end{array}\right], \\
\hat{\Sigma}_{f k} \triangleq\left[\begin{array}{c}
0 \\
\Sigma_{f k}
\end{array}\right], \quad \hat{\Sigma}_{h k} \triangleq\left[\begin{array}{c}
0 \\
\Sigma_{h k}
\end{array}\right], \quad \hat{B}_{k} \triangleq\left[\begin{array}{c}
0 \\
B_{k}
\end{array}\right], \\
\hat{H}_{k} \triangleq\left[\begin{array}{c}
0 \\
H_{k}
\end{array}\right], \quad \hat{E}_{k} \triangleq\left[\begin{array}{c}
0 \\
E_{k}
\end{array}\right], \quad \mathcal{L} \triangleq\left[\begin{array}{ll}
0 & L_{k}
\end{array}\right] .
\end{gathered}
$$

Next, defining a new vector $\eta_{k}$ by

$$
\begin{aligned}
\eta_{k} & \triangleq\left[\begin{array}{llllll}
\zeta_{k}^{\mathrm{T}} & \xi_{k}^{\mathrm{T}} & \vartheta_{k}^{\mathrm{T}} & \delta_{k}^{\mathrm{T}} & \rho_{k}^{\mathrm{T}} & \phi_{k}^{\mathrm{T}}
\end{array}\right]^{\mathrm{T}} \\
& =\left[\begin{array}{lllllll}
1 & e_{k}^{\mathrm{T}} & \xi_{k}^{\mathrm{T}} & \vartheta_{k}^{\mathrm{T}} & \delta_{k}^{\mathrm{T}} & \rho_{k}^{\mathrm{T}} & \phi_{k}^{\mathrm{T}}
\end{array}\right]^{\mathrm{T}},
\end{aligned}
$$

we further express the filtering error dynamics in the following compact form:

$$
\left\{\begin{aligned}
\zeta_{k+1} & =\tilde{f}_{k} \eta_{k}+\omega_{k} \tilde{h}_{k} \eta_{k} \\
\tilde{z}_{k} & =\mathcal{L}_{k} \zeta_{k}
\end{aligned}\right.
$$

where

$$
\begin{aligned}
& \tilde{f}_{k} \triangleq\left[\begin{array}{llllll}
\hat{f}_{k} & \mathcal{B}_{k} & \hat{\Sigma}_{f k} & 0 & 0 & \hat{H}_{k}
\end{array}\right], \quad \mathcal{B}_{k} \triangleq\left[\begin{array}{lll}
\hat{B}_{k} & 0
\end{array}\right], \\
& \tilde{h}_{k} \triangleq\left[\begin{array}{llllll}
\hat{h}_{k} & \mathcal{E}_{k} & 0 & \hat{\Sigma}_{f k} & 0 & 0
\end{array}\right], \quad \mathcal{E}_{k} \triangleq\left[\begin{array}{lll}
\hat{E}_{k} & 0
\end{array}\right] .
\end{aligned}
$$

In virtue of the RLMI approach, the following theorem presents a sufficient condition for the filtering error system (6) to satisfy the required $H_{\infty}$ performance specification.

Theorem 1: Let $\gamma>0, \Gamma>0$ and $\left\{F_{k}, H_{k}\right\}_{0 \leq k \leq N}$ be given. If there exist a sequence of positive definite matrices $\left\{P_{k}\right\}_{0 \leq k \leq N+1}$ with $P_{0} \leq \gamma^{2} \bar{\Gamma}(\bar{\Gamma} \triangleq \operatorname{diag}\{0, \Gamma\})$, sequences of positive scalars $\left\{\tau_{1 k}, \tau_{2 k}, \tau_{3 k}, \tau_{4 k}\right\}_{0 \leq k \leq N}$, a sequence of real value scalars $\left\{\varepsilon_{k}\right\}_{0 \leq k \leq N}$ such that

$$
\Omega_{k} \triangleq \bar{\Omega}_{k}-\sum_{i=1}^{4} \tau_{i k} \Omega_{i k}-\varepsilon_{k} \Omega_{\sigma k} \leq 0
$$

where

$$
\begin{aligned}
\bar{\Omega}_{k} \triangleq & \tilde{f}_{k}^{\mathrm{T}} P_{k+1} \tilde{f}_{k}+\alpha^{2} \tilde{h}_{k}^{\mathrm{T}} P_{k+1} \tilde{h}_{k} \\
& +\operatorname{diag}\left\{-P_{k}+\mathcal{L}_{k}^{\mathrm{T}} \mathcal{L}_{k},-\gamma^{2} I, 0,0,0,0\right\}, \\
\Omega_{1 k} \triangleq & \operatorname{diag}\{0,-I, 0, I, 0,0,0\} \\
\Omega_{2 k} \triangleq & \operatorname{diag}\{0,-I, 0,0, I, 0,0\}, \\
\Omega_{3 k} \triangleq & \operatorname{diag}\{0,-I, 0,0,0, I, 0\}, \\
\Omega_{4 k} \triangleq & \operatorname{diag}\{0,0,0,0,0,0, I\} \\
& -\mathcal{I}^{\mathrm{T}} U_{k} \tilde{g}_{k}-\tilde{g}_{k}^{\mathrm{T}} U_{k}^{\mathrm{T}} \mathcal{I}+\tilde{g}_{k}^{\mathrm{T}} \Lambda_{k} \tilde{g}_{k}, \\
\Omega_{\sigma k} \triangleq & \operatorname{diag}\left\{-\sigma_{k+1}+\lambda \sigma_{k}, 0,0,0,0,0,0\right\} \\
& +\tilde{g}_{k}^{\mathrm{T}} R_{k} \tilde{g}_{k},
\end{aligned}
$$

then the desired $H_{\infty}$ specification defined in (7) is achieved.

Proof: Defining $V_{k} \triangleq \zeta_{k}^{\mathrm{T}} P_{k} \zeta_{k}$, we have along the solution of (11) that

$$
\begin{aligned}
\Delta_{k} & =\mathbb{E}\left\{V_{k+1} \mid \eta_{k}\right\}-V_{k} \\
& =\mathbb{E}\left\{\zeta_{k+1}^{\mathrm{T}} P_{k+1} \zeta_{k+1}\right\}-\zeta_{k}^{\mathrm{T}} P_{k} \zeta_{k}
\end{aligned}
$$

$$
\begin{aligned}
= & \mathbb{E}\left\{\left(\tilde{f}_{k} \eta_{k}+\omega_{k} \tilde{h}_{k} \eta_{k}\right)^{\mathrm{T}} P_{k+1}\left(\tilde{f}_{k} \eta_{k}+\omega_{k} \tilde{h}_{k} \eta_{k}\right)\right\} \\
& -\zeta_{k}^{\mathrm{T}} P_{k} \zeta_{k} \\
= & \eta_{k}^{\mathrm{T}}\left(\tilde{f}_{k}^{\mathrm{T}} P_{k+1} \tilde{f}_{k}+\alpha^{2} \tilde{h}_{k}^{\mathrm{T}} P_{k+1} \tilde{h}_{k}\right) \eta_{k} \\
& -\eta_{k}^{\mathrm{T}} \operatorname{diag}\left\{P_{k}, 0,0,0,0,0\right\} \eta_{k} .
\end{aligned}
$$

Adding zero term $\tilde{z}_{k}^{\mathrm{T}} \tilde{z}_{k}-\gamma^{2} \xi_{k}^{\mathrm{T}} \xi_{k}-\left(\tilde{z}_{k}^{\mathrm{T}} \tilde{z}_{k}-\gamma^{2} \xi_{k}^{\mathrm{T}} \xi_{k}\right)$ to both sides of (19) yields

$$
\begin{aligned}
& \Delta_{k}+\tilde{z}_{k}^{\mathrm{T}} \tilde{z}_{k}-\gamma^{2} \xi_{k}^{\mathrm{T}} \xi_{k}-\left(\tilde{z}_{k}^{\mathrm{T}} \tilde{z}_{k}-\gamma^{2} \xi_{k}^{\mathrm{T}} \xi_{k}\right) \\
= & \Delta_{k}+\left(\mathcal{L}_{k} \zeta_{k}\right)^{\mathrm{T}} \mathcal{L}_{k} \zeta_{k}-\eta_{k} \operatorname{diag}\left\{0, \gamma^{2} I, 0,0,0,0\right\} \eta_{k} \\
& -\left(\tilde{z}_{k}^{\mathrm{T}} \tilde{z}_{k}-\gamma^{2} \xi_{k}^{\mathrm{T}} \xi_{k}\right) \\
= & \Delta_{k}+\eta_{k} \operatorname{diag}\left\{\mathcal{L}_{k}^{\mathrm{T}} \mathcal{L}_{k},-\gamma^{2} I, 0,0,0,0\right\} \eta_{k} \\
& -\left(\tilde{z}_{k}^{\mathrm{T}} \tilde{z}_{k}-\gamma^{2} \xi_{k}^{\mathrm{T}} \xi_{k}\right) \\
= & \eta_{k}^{\mathrm{T}} \bar{\Omega}_{k} \eta_{k}-\left(\tilde{z}_{k}^{\mathrm{T}} \tilde{z}_{k}-\gamma^{2} \xi_{k}^{\mathrm{T}} \xi_{k}\right)
\end{aligned}
$$

where $\bar{\Omega}_{k}$ is defined in (13).

On the other hand, bearing in mind the definition of $\vartheta_{k}$ and noting $\left\|\Delta_{f k}\right\| \leq 1$, we obtain

$$
\vartheta_{k}^{\mathrm{T}} \vartheta_{k}=e_{k}^{\mathrm{T}} \Delta_{f k}^{\mathrm{T}} \Delta_{f k} e_{k} \leq e_{k}^{\mathrm{T}} e_{k}
$$

which can be equivalently expressed by

$$
\eta_{k}^{\mathrm{T}} \Omega_{1 k} \eta_{k} \leq 0
$$

where $\Omega_{1 k}$ is defined in (14).

Similarly, it is inferred from $\delta_{k}=\Delta_{h k} e_{k}, \rho_{k}=\Delta_{g k} e_{k}$ (with $\left\|\Delta_{h k}\right\| \leq 1$ and $\left\|\Delta_{g k}\right\| \leq 1$ ) that

$$
\eta_{k}^{\mathrm{T}} \Omega_{2 k} \eta_{k} \leq 0, \quad \eta_{k}^{\mathrm{T}} \Omega_{3 k} \eta_{k} \leq 0
$$

where $\Omega_{2 k}$ and $\Omega_{3 k}$ are defined in (15) and (16), respectively.

Let us proceed to deal with the saturation function $\phi_{k}$. It is known from (4) that

$$
\left(\phi_{k}-\Lambda r_{k}\right)^{\mathrm{T}}\left(\phi_{k}-r_{k}\right) \leq 0
$$

which, by noticing $U_{k} \triangleq \frac{1}{2}\left(\Lambda_{k}+I\right)$, can be equivalently described by

$$
\phi_{k}^{\mathrm{T}} \phi_{k}-\phi_{k}^{\mathrm{T}} U_{k} r_{k}-r_{k}^{\mathrm{T}} U_{k}^{\mathrm{T}} \phi_{k}+r_{k}^{\mathrm{T}} \Lambda_{k} r_{k} \leq 0 .
$$

It is easily known that

$$
\begin{aligned}
r_{k} & =y_{k}-\hat{y}_{k} \\
& =g\left(\hat{x}_{k}\right)+\Psi_{k} e_{k}+\Sigma_{g k} \Delta_{g k} e_{k}+D_{k} \nu_{k}-g\left(\hat{x}_{k}\right) \\
& =\Psi_{k} e_{k}+\Sigma_{g k} \rho_{k}+D_{k} \nu_{k} \\
& =\tilde{g}_{k} \eta_{k}
\end{aligned}
$$

where $\tilde{g}_{k}$ is defined previously. Consequently, (25) can be described by

$$
\begin{aligned}
& \eta_{k}^{\mathrm{T}} \operatorname{diag}\{0,0,0,0,0,0, I\} \eta_{k}-\eta_{k}^{\mathrm{T}} \mathcal{I}^{\mathrm{T}} U_{k} \tilde{g}_{k} \eta_{k} \\
& -\eta_{k}^{\mathrm{T}} \tilde{g}_{k}^{\mathrm{T}} U_{k}^{\mathrm{T}} \mathcal{I} \eta_{k}+\eta_{k}^{\mathrm{T}} \tilde{g}_{k}^{\mathrm{T}} \Lambda_{k} \tilde{g}_{k} \eta_{k} \leq 0
\end{aligned}
$$

or, equivalently,

$$
\eta_{k}^{\mathrm{T}} \Omega_{4 k} \eta_{k} \leq 0
$$

where $\Omega_{4 k}$ is defined in (17).

From the constraint (3) imposed on the saturation level, we have

$$
\sigma_{k+1}=\lambda \sigma_{k}+\left(y_{k}-\hat{y}_{k}\right)^{\mathrm{T}} R_{k}\left(y_{k}-\hat{y}_{k}\right)
$$

which, in terms of $\eta_{k}$, can be expressed by

$$
\eta_{k}^{\mathrm{T}}\left(\operatorname{diag}\left\{-\sigma_{k+1}+\lambda \sigma_{k}, 0,0,0,0,0,0\right\}+\tilde{g}_{k}^{\mathrm{T}} R_{k} \tilde{g}_{k}\right) \eta_{k}=0
$$

or, equivalently,

$$
\eta_{k}^{\mathrm{T}} \Omega_{\sigma k} \eta_{k}=0
$$


where $\Omega_{\sigma k}$ is defined in (18).

According to Lemma 1, under the conditions of this theorem, we obtain $\bar{\Omega}_{k} \leq 0$. Subsequently, summing up both sides of (20) with respect to $k$ from 0 to $N$ leads to

$$
\sum_{k=0}^{N} \Delta_{k}=\sum_{k=0}^{N} \eta_{k}^{\mathrm{T}} \bar{\Omega}_{k} \eta_{k}-\sum_{k=0}^{N} \mathbb{E}\left\{\tilde{z}_{k}^{\mathrm{T}} \tilde{z}_{k}-\gamma^{2} \xi_{k}^{\mathrm{T}} \xi_{k}\right\}
$$

or,

$$
\begin{aligned}
& \sum_{k=0}^{N} \mathbb{E}\left\{\left\|\tilde{z}_{k}\right\|^{2}\right\}-\sum_{k=0}^{N} \gamma^{2}\left\|\xi_{k}\right\|^{2} \\
= & \sum_{k=0}^{N} \eta_{k}^{\mathrm{T}} \bar{\Omega}_{k} \eta_{k}-\sum_{k=0}^{N} \Delta_{k} \\
= & \sum_{k=0}^{N} \eta_{k}^{\mathrm{T}} \bar{\Omega}_{k} \eta_{k}-\eta_{N+1}^{\mathrm{T}} P_{k+1} \eta_{k+1}+\eta_{0}^{\mathrm{T}} P_{0} \eta_{0} .
\end{aligned}
$$

Noting that $\bar{\Omega}_{k} \leq 0, P_{k+1}>0$ and $P_{0} \leq \gamma^{2} \bar{\Gamma}$, we arrive at

$$
\sum_{k=0}^{N} \mathbb{E}\left\{\left\|\tilde{z}_{k}\right\|^{2}\right\} \leq \sum_{k=0}^{N} \gamma^{2}\left\|\xi_{k}\right\|^{2}+\gamma^{2} e_{0}^{\mathrm{T}} \Gamma e_{0}
$$

which indicates that the $H_{\infty}$ specification is achieved. The proof is now complete.

Remark 6: Theorem 1 provides a sufficient condition for the addressed filtering problem such that the output estimation error satisfies the prespecified disturbance attenuation level. By virtue of a set of recursive linear matrix inequalities, the required $H_{\infty}$ criterion can be guaranteed if the proposed set of RLMIs is feasible.

\section{B. Envelope-constraint in probability $\mathbf{p}$}

Before carrying out the analysis on the envelope-constraint with a desired probability $\mathbf{p}$, we first propose the following lemma that will be playing a vital role in the establishment of our main results.

Lemma 4: Let the filtering parameters $\left\{F_{k}, H_{k}\right\}_{0 \leq k \leq N}$ be given. If there exist a family of positive definite matrices $\left\{Q_{k}\right\}_{0 \leq k \leq N+1}$, sequences of positive scalars $\left\{\epsilon_{1 k}, \epsilon_{2 k}, \epsilon_{3 k}, \epsilon_{4 k}, \epsilon_{5 k}\right\}_{0 \leq k \leq N}$ and a sequence of real value scalars $\left\{\beta_{k}\right\}_{0 \leq k \leq N}$ satisfying the following recursive linear matrix inequality:

$$
\left[\begin{array}{ccc}
\bar{\Xi}_{k} & \mathscr{F}_{k}^{\mathrm{T}} & \alpha \mathscr{H}_{k}^{\mathrm{T}} \\
* & -Q_{k+1} & 0 \\
* & * & -Q_{k+1}
\end{array}\right] \leq 0
$$

where

$$
\begin{aligned}
& \bar{\Xi}_{k} \triangleq-\operatorname{diag}\{1,0,0,0,0,0\}-\sum_{j=1}^{5} \epsilon_{j k} \Xi_{j k}-\beta_{k} \Xi_{\sigma k}, \\
& \mathscr{F}_{k} \triangleq\left[f\left(\hat{x}_{k}\right)-F_{k} \hat{x}_{k}+\tilde{B}_{k} \xi_{k}^{\circ} \quad \Phi_{k} \Theta_{k}\right. \\
& \left.\Sigma_{f k} \quad 0 \quad 0 \quad-H_{k}\right], \\
& \mathscr{H}_{k} \triangleq\left[\begin{array}{llllll}
h\left(\hat{x}_{k}\right)+\tilde{E}_{k} \xi_{k}^{\circ} & \Pi_{k} \Theta_{k} & 0 & \Sigma_{f k} & 0 & 0
\end{array}\right], \\
& \mathscr{G}_{k} \triangleq\left[\begin{array}{llllll}
\mathcal{D}_{k} \xi_{k}^{\circ} & \Psi_{k} \Theta_{k} & 0 & 0 & \Sigma_{g k} & 0
\end{array}\right], \\
& \tilde{B}_{k} \triangleq\left[\begin{array}{cc}
B_{k} & 0
\end{array}\right], \quad \tilde{E}_{k} \triangleq\left[\begin{array}{ll}
E_{k} & 0
\end{array}\right] \text {, } \\
& \Xi_{q} \triangleq \operatorname{diag}\{-1, I, 0,0,0,0\} \text {, } \\
& \Xi_{2 k} \triangleq \operatorname{diag}\left\{0,-\Theta_{k}^{\mathrm{T}} \Theta_{k}, I, 0,0,0\right\} \text {, } \\
& \Xi_{3 k} \triangleq \operatorname{diag}\left\{0,-\Theta_{k}^{\mathrm{T}} \Theta_{k}, 0, I, 0,0\right\} \text {, } \\
& \Xi_{4 k} \triangleq \operatorname{diag}\left\{0,-\Theta_{k}^{\mathrm{T}} \Theta_{k}, 0,0, I, 0\right\} \text {, } \\
& \Xi_{5 k} \triangleq \operatorname{diag}\{0,0,0,0,0, I\} \\
& -\overline{\mathcal{I}}^{\mathrm{T}} U_{k} \mathscr{G}_{k}-\mathscr{G}_{k}^{\mathrm{T}} U_{k}^{\mathrm{T}} \overline{\mathcal{I}}+\mathscr{G}_{k}^{\mathrm{T}} \Lambda_{k} \mathscr{G}_{k}, \\
& \overline{\mathcal{I}} \triangleq\left[\begin{array}{llllll}
0 & 0 & 0 & 0 & 0 & I
\end{array}\right] \text {, }
\end{aligned}
$$

$$
\Xi_{\sigma k} \triangleq \operatorname{diag}\left\{-\sigma_{k+1}+\lambda \sigma_{k}, 0,0,0,0,0\right\}+\mathscr{G}_{k}^{\mathrm{T}} R_{k} \mathscr{G}_{k},
$$

then the following inequality holds for all $k \in[0, N]$ :

$$
\mathbb{E}\left\{\left(x_{k}-\hat{x}_{k}\right)^{\mathrm{T}} Q_{k}^{-1}\left(x_{k}-\hat{x}_{k}\right)\right\} \leq 1 .
$$

Proof: Applying input $\xi_{k}^{\circ}$ to filtering error system (6) leads to

$$
\begin{aligned}
e_{k+1}= & f\left(\hat{x}_{k}\right)+\Phi_{k} e_{k}+\Sigma_{f k} \vartheta_{k}+B_{k} \mu_{k} \\
& +\left(h\left(\hat{x}_{k}\right)+\Pi_{k} e_{k}+\Sigma_{h k} \delta_{k}+E_{k} \mu_{k}\right) \omega_{k} \\
& -F_{k} \hat{x}_{k}-H_{k} \phi_{k} .
\end{aligned}
$$

The rest of the proof is conducted by induction. First, when $k=0$, it is inferred from the zero-initial condition and $\hat{x}_{0}=0$ that

$$
\mathbb{E}\left\{\left(x_{0}-\hat{x}_{0}\right)^{\mathrm{T}} Q_{0}^{-1}\left(x_{0}-\hat{x}_{0}\right)\right\} \leq 1 .
$$

Next, assume that the following inequality holds at the time step $k$ :

$$
\mathbb{E}\left\{\left(x_{k}-\hat{x}_{k}\right)^{\mathrm{T}} Q_{k}^{-1}\left(x_{k}-\hat{x}_{k}\right)\right\} \leq 1 .
$$

Then, we only need to demonstrate that, at time step $k+1$, under the conditions given in this lemma, the following inequality holds:

$$
\mathbb{E}\left\{\left(x_{k+1}-\hat{x}_{k+1}\right)^{\mathrm{T}} Q_{k+1}^{-1}\left(x_{k+1}-\hat{x}_{k+1}\right)\right\} \leq 1 .
$$

To this end, since (36) is true, we have from [13] that there exists a vector $q_{k}$ satisfying $\mathbb{E}\left\{q_{k}^{\mathrm{T}} q_{k}\right\} \leq 1$ such that

$$
x_{k}=\hat{x}_{k}+\Theta_{k} q_{k}
$$

where $\Theta_{k}$ is a factorization of $Q_{k}$ (i.e., $Q_{k}=\Theta_{k} \Theta_{k}^{\mathrm{T}}$ ).

By using (38), the filtering error dynamics (34) driven by the input $\xi_{k}^{\circ}$ can be described by

$$
\begin{aligned}
e_{k+1}= & f\left(\hat{x}_{k}\right)+\Phi_{k} \Theta_{k} q_{k}+\tilde{B}_{k} \xi_{k}^{\circ} \\
& +\left(h\left(\hat{x}_{k}\right)+\Pi_{k} \Theta_{k} q_{k}+\Sigma_{h k} \delta_{k}+\tilde{E}_{k} \xi_{k}^{\circ}\right) \omega_{k} \\
& -F_{k} \hat{x}_{k}-H_{k} \phi_{k}
\end{aligned}
$$

which can be further expressed as

$$
e_{k+1}=\mathscr{F}_{k} \psi_{k}+\mathscr{H}_{k} \omega_{k} \psi_{k}
$$

where

$$
\psi_{k} \triangleq\left[\begin{array}{llllll}
1 & q_{k}^{\mathrm{T}} & \vartheta_{k}^{\mathrm{T}} & \delta_{k}^{\mathrm{T}} & \rho_{k}^{\mathrm{T}} & \phi_{k}^{\mathrm{T}}
\end{array}\right]^{\mathrm{T}} .
$$

Consequently, we have

$$
\begin{aligned}
& \mathbb{E}\left\{\left(x_{k+1}-\hat{x}_{k+1}\right)^{\mathrm{T}} Q_{k+1}^{-1}\left(x_{k+1}-\hat{x}_{k+1}\right)\right\} \\
= & \mathbb{E}\left\{\left(\mathscr{F}_{k} \psi_{k}+\mathscr{H}_{k} \omega_{k} \psi_{k}\right)^{\mathrm{T}} Q_{k+1}^{-1}\left(\mathscr{F}_{k} \psi_{k}+\mathscr{H}_{k} \omega_{k} \psi_{k}\right)\right\} \\
= & \psi_{k}^{\mathrm{T}} \mathscr{F}_{k}^{\mathrm{T}} Q_{k+1}^{-1} \mathscr{F}_{k} \psi_{k}+\alpha^{2} \psi_{k}^{\mathrm{T}} \mathscr{H}_{k}^{\mathrm{T}} Q_{k+1}^{-1} \mathscr{H}_{k} \psi_{k} \\
= & \psi_{k}^{\mathrm{T}}\left(\mathscr{F}_{k}^{\mathrm{T}} Q_{k+1}^{-1} \mathscr{F}_{k}+\alpha^{2} \mathscr{H}_{k}^{\mathrm{T}} Q_{k+1}^{-1} \mathscr{H}_{k}\right) \psi_{k} .
\end{aligned}
$$

Similar to the proof of Theorem 1 , we have from $\mathbb{E}\left\{q_{k}^{\mathrm{T}} q_{k}\right\} \leq 1$ that

$$
\mathbb{E}\left\{\psi_{k}^{\mathrm{T}} \Xi_{q} \psi_{k}\right\} \leq 0
$$

It is not difficult to infer from $\vartheta_{k}=\Delta_{f k} \Theta_{k} q_{k}$ and $\left\|\Delta_{f k}\right\| \leq 1$ that

$$
\vartheta_{k}^{\mathrm{T}} \vartheta_{k}=q_{k}^{\mathrm{T}} \Theta_{k}^{\mathrm{T}} \Delta_{f k}^{\mathrm{T}} \Delta_{f k} \Theta_{k} q_{k} \leq q_{k}^{\mathrm{T}} \Theta_{k}^{\mathrm{T}} \Theta_{k} q_{k}
$$

which can be equivalently expressed as

$$
\psi_{k}^{\mathrm{T}} \Xi_{2 k} \psi_{k} \leq 0
$$

Moreover, along similar lines, we acquire

$$
\psi_{k}^{\mathrm{T}} \Xi_{3 k} \psi_{k} \leq 0
$$

and

$$
\psi_{k}^{\mathrm{T}} \Xi_{4 k} \psi_{k} \leq 0
$$


Let us now proceed to deal with the saturation constraint (4). First, $r_{k}$ can be re-written as follows:

$$
\begin{aligned}
r_{k} & =y_{k}-\hat{y}_{k} \\
& =g\left(\hat{x}_{k}\right)+\Psi_{k} \Theta_{k} q_{k}+\Sigma_{g k} \rho_{k}+\mathcal{D}_{k} \xi_{k}^{\circ}-g\left(\hat{x}_{k}\right) \\
& =\mathscr{G}_{k} \psi_{k} .
\end{aligned}
$$

Then, inequality (4) can be equivalently described by

$$
\psi_{k}^{\mathrm{T}} \Xi_{5 k} \psi_{k} \leq 0
$$

Likewise, we obtain from the saturation level constraint (3) that

$$
\psi_{k}^{\mathrm{T}} \Xi_{\sigma k} \psi_{k}=0
$$

According to the Schur Complement Lemma, we know from (32) that

$$
\begin{gathered}
\mathscr{F}_{k}^{\mathrm{T}} Q_{k+1}^{-1} \mathscr{F}_{k}+\alpha^{2} \mathscr{H}_{k}^{\mathrm{T}} Q_{k+1}^{-1} \mathscr{H}_{k} \\
-\operatorname{diag}\{1,0,0,0,0,0\} \\
-\sum_{j=1}^{5} \epsilon_{j k} \Xi_{j k}-\beta_{k} \Xi_{\sigma k} \leq 0
\end{gathered}
$$

which, in combination with Lemma 1 , leads to

$$
\begin{array}{r}
\psi_{k}^{\mathrm{T}}\left(\mathscr{F}_{k}^{\mathrm{T}} Q_{k+1}^{-1} \mathscr{F}_{k}+\alpha^{2} \mathscr{H}_{k}^{\mathrm{T}} Q_{k+1}^{-1} \mathscr{H}_{k}\right. \\
-\operatorname{diag}\{1,0,0,0,0,0\}) \psi_{k} \leq 0 .
\end{array}
$$

Therefore, we arrive at

$$
\mathbb{E}\left\{\left(x_{k+1}-\hat{x}_{k+1}\right)^{\mathrm{T}} Q_{k+1}^{-1}\left(x_{k+1}-\hat{x}_{k+1}\right)\right\} \leq 1 .
$$

The proof is now complete.

Theorem 2: Let the filtering gains $\left\{F_{k}, H_{k}\right\}_{0 \leq k \leq N}$, the desired output $\left\{\varphi_{k}\right\}_{0 \leq k \leq N}$, the tolerance band $\left\{\chi_{k}\right\}_{0 \leq k \leq N}$ and the desired probability $\mathbf{p}$ be given. If there exist a family of positive definite matrices $\left\{Q_{k}\right\}_{0 \leq k \leq N+1}$, sequences of positive scalars $\left\{\epsilon_{1, k}, \epsilon_{2, k}, \epsilon_{3, k}, \epsilon_{4, k}, \epsilon_{5, k}, \varsigma_{k}\right\}_{0 \leq k \leq N}$ and a sequence of real value scalars $\left\{\beta_{k}\right\}_{0 \leq k \leq N}$ satisfying the following recursive linear matrix inequalities:

$$
\begin{gathered}
{\left[\begin{array}{ccc}
\bar{\Xi}_{k} & \mathscr{F}_{k}^{\mathrm{T}} & \alpha \mathscr{H}_{k}^{\mathrm{T}} \\
* & -Q_{k+1} & 0 \\
* & * & -Q_{k+1}
\end{array}\right] \leq 0,} \\
{\left[\begin{array}{cc}
-\tilde{\chi}_{k}+\varsigma_{k}+\varphi_{k}^{2} & -\varphi_{k}^{\mathrm{T}} L_{k} \Theta_{k} \\
* & -\varsigma_{k} I+\Theta_{k}^{\mathrm{T}} L_{k}^{\mathrm{T}} L_{k} \Theta_{k}
\end{array}\right] \leq 0}
\end{gathered}
$$

where

$$
\tilde{\chi}_{k} \triangleq \chi_{k}^{2}(1-\mathbf{p})
$$

then the envelope-constraint in probability $\mathbf{p}$ defined in (9) is achieved.

Proof: Since (53) is satisfied, we know from Theorem 2 that, over the horizon $k \in[0, N]$, the errors $e_{k}$ and $\tilde{z}_{k}^{\circ}$ can be described by $e_{k}=\Theta_{k} q_{k}$ and $\tilde{z}_{k}^{\circ}=L_{k} \Theta_{k} q_{k}$, respectively, where $\Theta_{k}$ is a factorization of $Q_{k}$. By defining $\varpi_{k} \triangleq\left[\begin{array}{cc}1 & q_{k}^{\mathrm{T}}\end{array}\right]^{\mathrm{T}}$, we have

$$
\tilde{z}_{k}^{\circ}-\varphi_{k}=\left[\begin{array}{ll}
-\varphi_{k} & L_{k} \Theta_{k}
\end{array}\right] \varpi_{k} .
$$

Consequently, the following is true:

$$
\left(\tilde{z}_{k}^{\circ}-\varphi_{k}\right)^{2}-\tilde{\chi}_{k}=\varpi_{k}^{\mathrm{T}} \Upsilon_{k} \varpi_{k}
$$

where

$$
\Upsilon_{k} \triangleq\left[\begin{array}{c}
-\varphi_{k} \\
\Theta_{k}^{\mathrm{T}} L_{k}^{\mathrm{T}}
\end{array}\right]\left[\begin{array}{ll}
-\varphi_{k} & L_{k} \Theta_{k}
\end{array}\right]+\operatorname{diag}\left\{-\tilde{\chi}_{k}, 0\right\} .
$$

On the other hand, it is readily obtained from the condition (54) that

$$
\Upsilon_{k}-\varsigma_{k} \operatorname{diag}\{-1, I\} \leq 0
$$

which further implies

$$
\mathbb{E}\left\{\varpi_{k}^{\mathrm{T}} \Upsilon_{k} \varpi_{k}\right\}-\mathbb{E}\left\{\varsigma_{k} \varpi_{k}^{\mathrm{T}} \operatorname{diag}\{-1, I\} \varpi_{k}\right\} \leq 0 .
$$

Since it is inferred from $\mathbb{E}\left\{q_{k}^{\mathrm{T}} q_{k}\right\} \leq 1$ that

$$
\mathbb{E}\left\{\varpi_{k}^{\mathrm{T}} \operatorname{diag}\{-1, I\} \varpi_{k}\right\} \leq 0,
$$

we arrive at

$$
\mathbb{E}\left\{\left(\tilde{z}_{k}^{\circ}-\varphi_{k}\right)^{2}\right\} \leq \tilde{\chi}_{k} .
$$

Inequality (60) indicates that

$$
\mathbb{E}\left\{\left(\frac{1}{\chi_{k}^{2}}\left(\tilde{z}_{k}^{\circ}-\varphi_{k}\right)^{2}\right\} \leq 1-\mathbf{p}\right.
$$

which, according to Lemma 3 , is equivalent to

$$
\mathbb{P}\left\{\left|\tilde{z}_{k}^{\circ}-\varphi_{k}\right| \leq \chi_{k}\right\} \geq \mathbf{p} .
$$

The proof is now complete.

\section{Filter Design}

Having analyzed the $H_{\infty}$ performance and envelope-constraint in probability $\mathbf{p}$ in the previous subsections, we are now ready to give the solution to the probability-guaranteed envelope-constrained $H_{\infty}$ filter design problem for the addressed nonlinear time-varying system. The solvability of the formulated multi-objective filtering problem is presented in terms of the feasibility of a series of recursive linear matrix inequalities.

Theorem 3: Let $\gamma>0, \Gamma>0,\left\{\varphi_{k}, \chi_{k}\right\}_{0 \leq k \leq N}$ and $0<\mathbf{p}<1$ be given. If there exist sequences of positive definite matrices $\left\{P_{k}, Q_{k}\right\}_{0 \leq k \leq N+1}$ with $P_{0} \leq \gamma^{2} \bar{\Gamma}(\bar{\Gamma} \triangleq \operatorname{diag}\{0, \Gamma\})$, sequences of real value matrices $\left\{F_{k}, H_{k}\right\}_{0 \leq k \leq N}$, sequences of positive scalars $\left\{\tau_{i k}, \epsilon_{j k}, \varsigma_{k}\right\}_{0 \leq k \leq N}(i=1,2,3,4 ; j=1,2,3,4,5)$, sequences of real value scalars $\left\{\varepsilon_{k}, \beta_{k}\right\}_{0 \leq k \leq N}$ such that

$$
\text { (12), (53) \& (54) }
$$

hold, then the design objectives $R 1$ and $R 2$ are satisfied simultaneously.

Proof: Based on the proofs of Theorems 1 and 2, the theorem can be proved immediately and therefore is omitted here.

In the following, an iterative algorithm is presented to compute the sequences of the filtering parameters $\left\{F_{k}, H_{k}\right\}_{0 \leq k \leq N}$ recursively.

Algorithm 1: Computational Algorithm for $\left\{F_{k}, H_{k}\right\}_{0 \leq k \leq N}$

1) Initialization: Set $k=0$. Set parameters $\left(\gamma, \Gamma, \mathrm{p},\left\{\varphi_{k}, \chi_{k}\right\}_{0 \leq k \leq N}\right)$. Choose $P_{0}$ satisfying $P_{0} \leq \gamma^{2} \Gamma$. Choose $Q_{0}$ and then factorize $Q_{0}$ appropriately to obtain the matrix $\Theta_{0}$.

2) Solve the RLMIs (12), (53) \& (54) for $\left\{F_{k}, H_{k}\right\}$. The matrices $P_{k}$ and $Q_{k}$ are also obtained. Then factorize $Q_{k}$ appropriately to obtain the matrix $\Theta_{k}$.

3) Set $k=k+1$. If $k>N$, exit. Otherwise, go to 2).

Remark 7: It follows from Theorem 3 that the desired filtering parameters could be a set if non-empty. An interesting issue would be to seek certain suboptimal solutions among the feasible set based on some criteria of engineering significance. It should be emphasized that, thanks to the flexibility of the provided framework, it is not difficult to design certain suboptimal filters via optimizing one index while fixing the other.

Remark 8: Notice that the RLMI algorithm proposed in this paper is based on an LMI approach. As discussed in [22], the computational 
complexity of an LMI system is bounded by $O(\mathscr{P} \mathscr{Q} 3 \log (\mathscr{U} / \varepsilon))$ where $\mathscr{P}$ represents the row size, $\mathscr{Q}$ stands for the number of scalar decision variables, $\mathcal{U}$ is a data-dependent scaling factor and $\varepsilon$ is relative accuracy set for algorithm. The complexity of our provided algorithm can then be easily computed by resorting to the formula developed in [22].

Remark 9: In this paper, a systematic investigation is initiated on the recursive filtering problem for nonlinear time-varying stochastic systems subject to possible measurement outliers. The main novelties of this paper are outlined as follows: 1) the filter structure is new in that an adaptive saturation function is introduced to resist the possible measurement outliers where the saturation level is recursively determined at each time step according to previous filtering errors; 2) the objectives of the transient performance are new in that both the $H_{\infty}$ specification and the envelope-constraint in probability are taken into account; and 3) the proposed algorithm is new in that a probabilistic constraint is introduced to ensure that the output of filtering error (stimulated by a specified input) is confined into a prescribed envelope with desired probability.

\section{NUMERICAL EXAMPLE}

In this section, we shall demonstrate the applicability of the proposed filtering algorithm via an illustrative example. First, for a vector $a \in \mathbb{R}^{2}$, denote by $a^{(1)}$ and $a^{(2)}$ the first and second entries of $a$, respectively.

Consider the nonlinear system (1) with the following parameters:

$$
\begin{aligned}
f\left(x_{k}\right) & =\left[\begin{array}{c}
\left.0.1 x^{(1)}+1.7 \sin \left(2 x^{(1)}+3\right)+0.1 \cos (1.2 k)\right) \\
0.6 x^{(2)}+0.2 \sin (k)
\end{array}\right], \\
g\left(x_{k}\right) & =\frac{x^{(1)}}{8}+\sin \left(x^{(2)}\right), h\left(x_{k}\right)=\left[\begin{array}{c}
0.9 x^{(1)}-0.9 \\
0.3 x^{(2)}+1.2
\end{array}\right], \\
B(k) & =\left[\begin{array}{c}
0.6 \sin (k)+2 \\
1.8+0.5 \cos (k+1)
\end{array}\right], D(k)=4 \sin (k)+2.5, \\
\xi^{\circ}(k) & =\left[\begin{array}{ll}
1 & 1
\end{array}\right]^{\mathrm{T}}, E(k)=\left[\begin{array}{ll}
0.9 & 1
\end{array}\right], L(k)=\left[\begin{array}{ll}
0.1 & 0.1
\end{array}\right], \\
\mu(k) & =3 \sin (2 k), \quad \nu(k)=\cos (2 k-1) /(2+3 k) .
\end{aligned}
$$

Assume that the initial values of the system state and its estimation are all zero, namely,

$$
x_{0}=\left[\begin{array}{ll}
0 & 0
\end{array}\right]^{\mathrm{T}}, \quad \hat{x}_{0}=\left[\begin{array}{ll}
0 & 0
\end{array}\right]^{\mathrm{T}} .
$$

Moreover, we choose the parameters as follows:

$$
\begin{aligned}
& \mathbf{p}=0.9, \quad \lambda=0.1, \quad R=0.9, \quad \alpha=0.3, \\
& \gamma=3, \quad \chi=3, \quad \varphi=-0.5, \\
& \Gamma=\operatorname{diag}_{2}\{0.1\}, Q_{0}=\operatorname{diag}_{2}\{1\}, P_{0}=\operatorname{diag}\{0,1,1\} .
\end{aligned}
$$

The simulation results are shown in Figs. $1-4$. To be specific, Fig. 1 depicts the estimation errors $e_{k}^{(1)}$ and $e_{k}^{(2)}$, while Fig. 2 shows the output filtering error $\tilde{z}_{k}^{\circ}$. From Fig. 2, we know that the filtering error $\tilde{z}_{k}^{\circ}$ is successfully constrained within the range determined by the prespecified upper and lower bounds. Therefore, it can be seen from Figs. 1-2 that the proposed filtering algorithm is applicable and performs quite well.

In order to show the ability of our design technique to attenuate the effects from the possible measurement outliers, we make comparisons of three cases with different saturation levels as follows: 1) the saturation level is adaptively changed (i.e., the mechanism proposed in this paper), denoted by ' $\sigma$-adaptive' in the simulation figures; 2 ) the case without saturation constraint (i.e., $\sigma_{k}=\infty$ ), denoted by ' $\sigma$ inf'; and 3) the saturation level is fixed as a constant value, denoted by ' $\sigma$-fixed'. The results are shown in Figs. 3-4, from which we can see that the proposed algorithm with adaptively changed saturation level can effectively reduce the impact from measurement outliers, thereby largely improving the estimation performances.

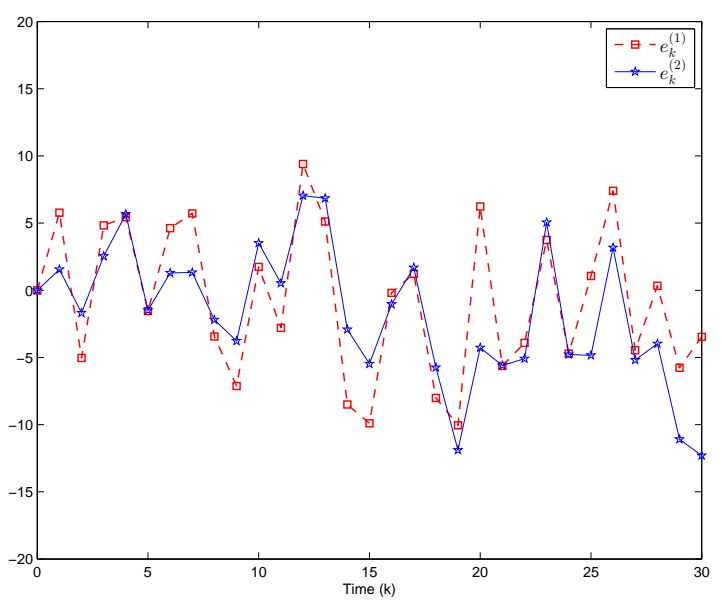

Fig. 1. The estimation errors $e_{k}^{(1)}$ and $e_{k}^{(2)}$.

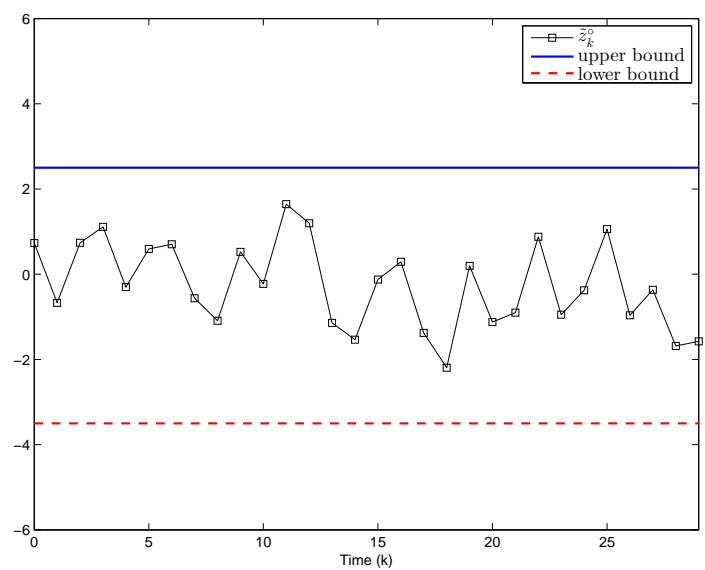

Fig. 2. The output of filtering error $\tilde{z}_{k}^{\circ}$ and the upper and lower bounds.

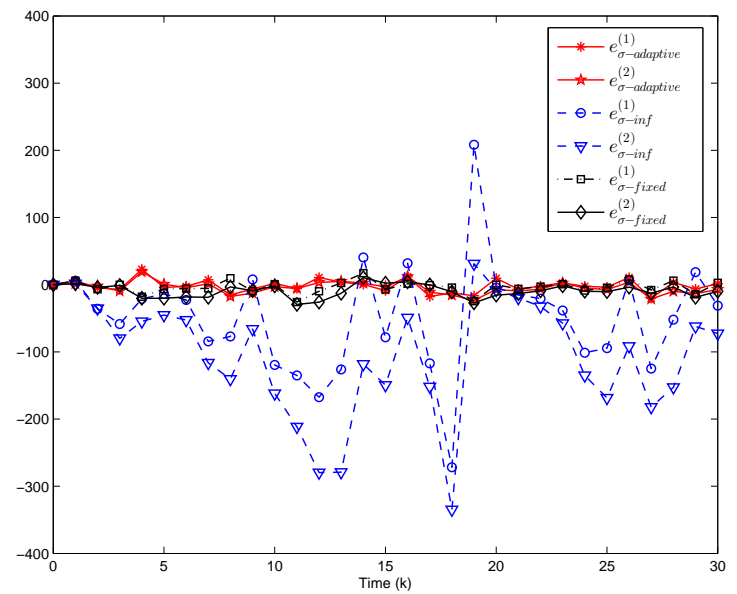

Fig. 3. The estimation error $e_{k}$ subject to different $\sigma_{k}$. 


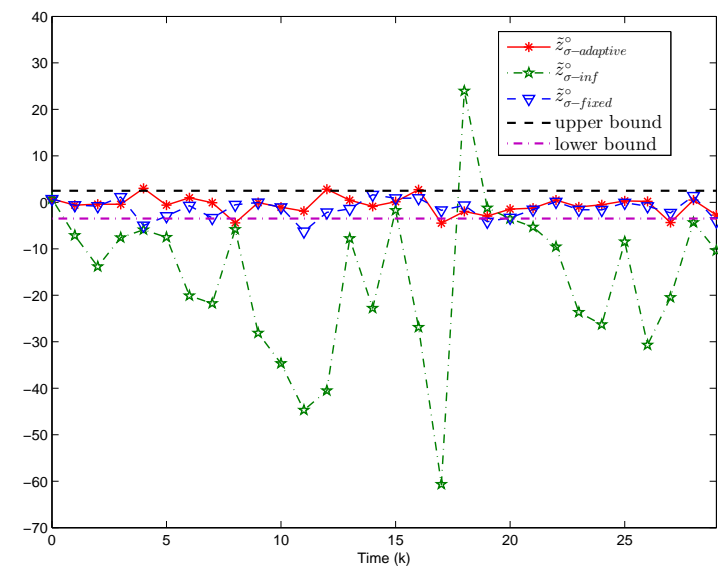

Fig. 4. The output of filtering error $\tilde{z}_{k}^{\circ}$ subject to different $\sigma_{k}$.

\section{Conclusions}

In this paper, we have investigated the filtering problem for nonlinear time-varying stochastic systems subject to possible measurement outliers. With the purpose of mitigating the effects from possible measurement outliers, a filter has been constructed with a saturated output injection where the saturation level is determined dynamically according to the filtering errors. Both the performance indices (i.e., the finite-horizon $H_{\infty}$ specification and the envelope-constraint criterion with a pre-determined probability) have been considered over the specified time interval. By resorting to the recursive linear matrix inequality approach, sufficient conditions have been derived for the existence of the desired filter guaranteeing desired performances. Finally, the proposed filtering algorithm has been verified via an illustrative numerical example. A possible research topic would be to extend the main results to more complicated systems with different filtering requirements such as distributed filtering [19], [20], minimum entropy filtering [29] and jamming-aware fusion [9]. Moreover, notice that the method used in this paper to deal with nonlinearities is Taylor expansion, which would inevitably result in linearization errors. Another potential research direction is to extend the obtained results for general nonlinear systems characterized by other methodologies such as T-S fuzzy model [17].

\section{REFERENCES}

[1] A. Alessandri and M. Awawdeh, Moving-horizon estimation with guaranteed robustness for discrete-time linear systems and measurements subject to outliers, Automatica, vol. 67, pp. 85-93, 2016.

[2] A. Alessandri and L. Zaccarian, Stubborn state observers for linear timeinvariant systems, Automatica, vol. 88, pp. 1-9, 2018.

[3] M. V. Basin and P. Cesar Rodriguez-Ramirez, Sliding-mode filter design for linear systems with unmeasured states, IEEE Transactions on Industrial Electronics, vol. 58, no. 8, pp. 3616-3622, 2011.

[4] S. Boyd, L. Ghaoui, E. Feron and V. Balakrishnan, Linear matrix inequalities in system and control theory, Philadelphia: SIAM Studies in Applied Mathematics, 1994.

[5] R. Caballero-Águila, A. Hermoso-Carazo and J. Linares-Pérez, Optimal state estimation for networked systems with random parameter matrices, correlated noises and delayed measurements, International Journal of General Systems, vol. 44, no. 2, pp. 142-154, 2015.

[6] G. Calafiore, Reliable localization using set-value nonlinear filters, IEEE Transactions on Systems, Man and Cybernetics-Part A: Systems and Humans, vol. 35, no. 2, pp. 189-197, 2005.

[7] Y. Chen, Z. Wang, S. Fei, and Q.-L. Han, Regional stabilization for discrete time-delay systems with actuator saturations via a delaydependent polytopic approach, IEEE Transactions on Automatic Control, vol. 64 , no. 3, pp. 1257-1264, 2019.
[8] A. Cantoni, B.-N. Vo and K. Teo, An introduction to envelope constrained filter design, Journal of Telecommunications and Information Technology, pp. 3-14, 2001.

[9] D. Ciuonzo, A. Aubry and V. Carotenuto, Rician MIMO channel- and jamming-aware decision fusion, IEEE Transactions on Signal Processing, vol. 65, no. 15, pp. 3866-3880, Aug. 2017.

[10] D. Ding, Z. Wang, B. Shen and H. Dong, Envelope-constrained $H_{\infty}$ filtering with fading measurements and randomly occurring nonlinearities: the finite horizon case, Automatica, vol. 55, pp. 37-45, 2015.

[11] G. A. Einicke and L. B. White, Robust extended Kalman filtering, IEEE Transactions on Signal Processing, vol. 47, no. 9, pp. 2596-2599, 1999.

[12] M. A. Gandhi and L. Mili, Robust Kalman filter based on a generalized maximum-likelihood-type estimator, IEEE Transactions on Automatic control, vol. 58, no. 5, pp. 2509-2520, 2010.

[13] L. El. Ghaoui and G. Calafiore, Robust filtering for discrete-time systems with bounded noise and parametric uncertainty, IEEE Transactions on Automatic Control, vol. 46, pp. 1084-1089, 2001.

[14] R. Gibbs, New Kalman filter and smoother consistency tests, Automatica, vol. 49, pp. 3141-3144, 2013.

[15] F. Han, G. Wei, D. Ding and Y. Song, Finite-horizon bounded $H_{\infty}$ synchronisation and state estimation for discrete-time complex networks: Local performance analysis, IET Control Theory \& Applications, vol. 11, no. 6, pp. 827-837, Apr. 2017.

[16] M. R. James, and I. R. Petersen, Nonlinear state estimation for uncertain systems with an integral constraint, IEEE Transactions on Signal Processing, vol. 46, no. 11, pp. 2926-2937, 1998.

[17] X. Liu, Z. Gao and A. Zhang, Observer-based fault estimation and tolerant control for stochastic Takagi-Sugeno fuzzy systems with Brownian parameter perturbations, Automatica, vol. 102, pp. 137-149, 2019.

[18] L. Ma, Z. Wang, Q.-L. Han and H.-K. Lam, Envelope-constrained $H_{\infty}$ filtering for nonlinear systems with quantization effects: The finite horizon case, Automatica, vol. 93, pp. 527-534, 2018.

[19] L. Ma, Z. Wang, H.-K. Lam, and N. Kyriakoulis, Distributed event-based set-membership filtering for a class of nonlinear systems with sensor saturations over sensor networks, IEEE Transactions on Cybernetics, vol. 47, no. 11, pp. 3772-3783, 2017.

[20] L. Ma, Z. Wang, Y. Liu and F. E. Alsaadi, Distributed filtering for nonlinear time-delay systems over sensor networks subject to multiplicative link noises and switching topology, International Journal of Robust and Nonlinear Control, vol. 29, no. 10, pp. 2941-2959, 2019.

[21] U. Shaked and N. Berman, $H_{\infty}$ nonlinear filtering of discrete-time processes, IEEE Transactions on Signal Processing, vol. 43, no. 9, pp. 2205-2209, 1995.

[22] B. Shen, Z. Wang and X. Liu, Bounded $H_{\infty}$ synchronization and state estimation for discrete time-varying stochastic complex networks over a finite horizon, IEEE Transactions on Neural Networks, vol. 22, no. 1, pp. 145-157, 2011.

[23] H. Tan, B. Shen, Y. Liu, A. Alsaedi and B. Ahmad, Event-triggered multi-rate fusion estimation for uncertain system with stochastic nonlinearities and colored measurement noises, Information Fusion, vol. 36, pp. 313-320, Jul. 2017.

[24] Z. Tan, Y. C. Soh and L. Xie, Envelope-constrained IIR filter design: An LMI $H_{\infty}$ optimization approach, Circuits Systems Signal Processing, vol. 19, no. 3, pp. 205-220, 2000.

[25] E. Tian, Z. Wang, L. Zou and D. Yue, Probability-constrained filtering for a class of nonlinear systems with improved static event-triggered communication, International Journal of Robust and Nonlinear Control, vol. 29, pp. 1484-1498, 2019.

[26] Z. Wang, B. Shen and X. Liu, $H_{\infty}$ filtering with randomly occurring sensor saturations and missing measurements, Automatica, vol. 48, pp. 556-562, 2012.

[27] L. Xie, Y. Soh, and C. E. de Souza, Robust Kalman filtering for uncertain discrete-time systems, IEEE Transactions on Automatic Control, vol. 39, no. 6, pp. 1310-1314, 1994.

[28] I. Yaesha, S. Boyarskia and U. Shaked, Probability-guaranteed robust $H_{\infty}$ performance analysis and state-feedback design, Systems and Control Letters, vol. 48, pp. 351-364, 2003.

[29] X. Yin, Q. Zhang, H. Wang and Z. Ding, RBFNN-based minimum entropy filtering for a class of stochastic nonlinear systems, IEEE Transactions on Automatic Control, vol. 65, no. 1, pp. 376-381, 2020.

[30] Z. Zang, A. Cantoni and K. Teo, Envelope-constrained IIR filter design via $H_{\infty}$ optimization methods. IEEE Transactionson Circuits and Systems-I, vol. 46, no. 6, pp. 649-653, 1999.

[31] X.-M. Zhang, Q.-L. Han, X. Ge, D. Ding, L. Ding, D. Yue and C. Peng, Networked control systems: A survey of trends and techniques, IEEE/CAA Journal of Automatica Sinica, vol. 7, no. 1, pp. 1-17, 2020. 